\title{
Allogeneic GM-CSF-secreting Lethally Irradiated Whole Melanoma Cell Vaccine
}

\author{
National Cancer Institute
}

\section{Source}

National Cancer Institute. Allogeneic GM-CSF-secreting Lethally Irradiated Whole

Melanoma Cell Vaccine. NCI Thesaurus. Code C98282.

An allogeneic cancer vaccine composed of lethally irradiated whole melanoma cancer cells that are genetically modified to secrete the immunostimulatory cytokine granulocyte-macrophage colony-stimulating factor (GM-CSF), with potential immunostimulating and antineoplastic activities. Upon intradermal injections, allogeneic GM-CSF-secreting lethally irradiated whole melanoma cell vaccine secretes GM-CSF. In turn, GM-CSF may stimulate the body's immune system against tumor cells by enhancing the activation of dendritic cells (DCs) and promoting antigen presentation to both B- and T-lymphocytes. In addition, GM-CSF promotes antibody-dependent cellular cytotoxicity (ADCC), and increases interleukin-2-mediated lymphokine-activated killer cell function. 